\title{
Trends in HPV-related oropharyngeal cancers in Singapore
}

\section{Dear Editor,}

We would like to highlight the burgeoning global epidemic of human papillomavirus (HPV)-associated oropharyngeal squamous cell carcinoma (OPSCC). OPSCC is commonly caused by smoking and alcohol, but incidence of HPV as the cause of OPSCC has been increasing, particularly in Western countries. A metaanalysis of 2,099 OPSCC cases in the US showed a significant increase in the prevalence of $\mathrm{HPV}$ association from $20.9 \%$ before 1990 to $65.4 \%$ after $2000 .{ }^{1}$ In contrast, there has been a relative paucity of data from Asian countries. A study from Hong Kong showed that $20.8 \%$ of OPSCC cases were associated with HPV. ${ }^{2}$ A study from Thailand of 110 cases of OPSCC diagnosed between 2010 and 2016 showed a prevalence of only $14.5 \% .^{3}$

Based on the Singapore Cancer Registry, there has been a steady increase in OPSCC incidence from 1993 to 2012 in both men and women over a 20-year period. ${ }^{4}$ However, to date, there is limited information on the prevalence of HPV-associated OPSCC in the multiracial population of Singapore. We aimed to examine whether rates of incidence in the Singapore population are high, similar to those seen in Western countries; or low, akin to those in Hong Kong and Thailand.

Between 1 January 2015 and 31 December 2019, a total of 68 patients were diagnosed with OPSCC at our institution, Tan Tock Seng Hospital, Singapore. HPV-positive status was determined by (1) positive immunohistochemical staining for $\mathrm{p} 16$, defined as strong and diffuse nuclear and cytoplasmic staining in over $70 \%$ of tumour cells in keeping with the American Joint Committee on Cancer recommendations (8th edition) $)^{5}$ and College of American Pathologists guidelines, ${ }^{6}$ and (2) classical histopathological features of non-keratinising carcinoma with varying degrees of basaloid differentiation.

The distribution of $\mathrm{p} 16$ status for the various OPSCC subsites is shown in Table 1, with $50 \%$ of tonsillar OPSCC and $47 \%$ of base of tongue OPSCC identified as $\mathrm{p} 16$-positive. The overall proportion of $\mathrm{p} 16$-positive OPSCC in our cohort is $41.2 \%$. This rate is similar to a Singapore study published in 2016, performed on 31 OPSCC cases with a p16-positivity rate of $45.2 \%$. $^{7}$ This indicates that the prevalence of HPV-associated OPSCC in Singapore is lower compared to its prevalence in the Western countries, and falls tentatively in between the rates of Western and other Asian countries.

Based on our hospital serving a population of approximately 1 million, the estimated incidence rates for HPV-associated OPSCC for the area served by our hospital (central Singapore) ranges from 0.30 to 0.81 per 100,000 persons per year from 2015 to 2019 . According to data from the US Centers for Disease Control and Prevention for HPV-associated cancers in the US from 2013 to 2017, HPV-associated OPSCC rates for American Caucasian and Asian populations are 5.5 and 1.3 per 100,000 persons, respectively approximately 6.8 and 1.6 times higher compared to the highest reported annual incidence in our population in 2019. ${ }^{8}$ The difference in the American data compared to ours may reflect an interplay of differences in genetic/ethnic factors as well as cultural differences in sexual practices. While it is impossible to determine the contributions of ethnicity versus cultural sexual behaviours from our data, there is suggestion that genetics and ethnicity may be independent risk factors given that Asian Americans-presumably with similar cultural behaviours to our population-have a lower incidence of HPV-associated OPSCC than white Caucasians. ${ }^{8}$

D'Souza et al. demonstrated that HPV-associated OPSCC is strongly related to sexual activity with multiple sexual partners and oral sex. ${ }^{9}$ In Singapore, the incidence of casual sex has markedly increased from $1.1 \%$ in 1989 to $17.4 \%$ in 2007 among heterosexuals in Singapore, with the majority (84\%) practising unprotected sex. ${ }^{10}$

Given the increased prevalence of high-risk sexual behaviours in Singapore, factoring in the approximately 40-year lag period between exposure and disease, it remains to be seen if HPV-associated OPSCC would reach epidemic status as is the case in Western countries. This is an important public health issue to be addressed and countermeasures have to be taken. In addition, the economic burden of HPV-associated OPSCC in countries with high prevalence has proven to be heavy. In France, the cost for hospitalisations related to HPV-associated OPSCC in 2007 was EUR138 million. ${ }^{11}$ Hence, it is important for Singapore to project the incidence of new cases in the ensuing years to prepare for increasing medical demand in hospitals and the economic cost in managing this burden.

Management of this burgeoning health problem should not only be reactive but preventive. The 
Table 1. Distribution of p16 status for the various oropharyngeal tumour subsites

\begin{tabular}{lcccccc}
\hline p16 status & \multicolumn{5}{c}{ Tumour subsite } \\
\cline { 2 - 6 } & Tonsil & Base of tongue & Soft palate & Posterior pharyngeal wall & Total \\
\hline Positive & 18 & 8 & 2 & 0 & 28 \\
\hline Negative & 18 & 9 & 7 & 6 & 40 \\
Total no. & 36 & 17 & 9 & 6 & 68 \\
\hline p16 positivity (\%) & 50 & 47 & 22.2 & 0 & 41.2 \\
\hline
\end{tabular}

Singapore Cancer Registry has started to monitor the HPV status of diagnosed OPSCC cases from 2018 onwards to observe the trend. In terms of education, the Health Promotion Board can play an active role to raise public awareness and promote primary prevention. The Singapore population should be educated to understand that OPSCC is a sexually transmitted disease and high-risk sexual behaviours include having casual sex with multiple partners.

In the US, the Food and Drug Administration has approved the use of Gardasil 9 vaccine (9-valent HPV vaccine) in both males and females (ages 9-45) for the prevention of oropharyngeal and other head and neck cancers caused by HPV. ${ }^{12}$ In Singapore, the National Childhood Immunisation Programme only recommends the vaccination of girls and women aged 9-26 years old for the prevention of cervical cancer. ${ }^{13}$ It is therefore important to monitor the prevalence trends of HPV-associated OPSCC in Singapore to formulate our own national vaccine strategy for this disease.

In conclusion, the prevalence of HPV-associated OPSCC in Singapore is currently in between that of Western and other Asian countries. Rising rates of HPV-associated OPSCC across the world suggest that this is an important health problem to consider in Singapore. Education of the Singapore population and vaccination may be important preventive measures. Hospitals in Singapore should work together with the Health Promotion Board and the Ministry of Health to formulate judicious health-related economic policies to address this burgeoning problem.

\section{REFERENCES}

1. Stein AP, Saha S, Yu M, et al. Prevalence of human papillomavirus in oropharyngeal squamous cell carcinoma in the United States across time. Chem Res Toxicol 2014;27:462-9.

2. Lam EW, Chan JY, Chan AB, et al. Prevalence, clinicopathological Characteristics, and outcome of human papillomavirus-associated oropharyngeal cancer in southern Chinese patients. Cancer Epidemiol Biomarkers Prev 2016;25:165-73.

3. Nopmaneepaisarn T, Tangjaturonrasme N, Rawangban W, et al. Low prevalence of p16-positive HPV-related head-neck cancers in Thailand: tertiary referral center experience. BMC Cancer 2019;19:1050.
4. Lam JO, Lim WY, Chow KY, et al. Incidence, Trends and Ethnic Differences of Oropharyngeal, Anal and Cervical Cancers: Singapore, 1968-2012. PLoS One 2015;10:e0146185.

5. Lydiatt WM, Patel SG, O'Sullivan B, et al. Head and Neck cancersmajor changes in the American Joint Committee on cancer eighth edition cancer staging manual. CA Cancer J Clin 2017;67:122-37.

6. Lewis JS Jr, Beadle B, Bishop JA, et al. Human Papillomavirus Testing in Head and Neck Carcinomas: Guideline From the College of American Pathologists. Arch Pathol Lab Med 2018;142:559-97.

7. Tan LS, Fredrik P, Ker L, et al. High-risk HPV genotypes and P16INK4a expression in a cohort of head and neck squamous cell carcinoma patients in Singapore. Oncotarget 2016;7:86730-9.

8. Centers for Disease Control and Prevention. Annual Number and Rate of HPV-Associated Cancers by Cancer Site, Sex, and Race and Ethnicity, United States, 2013-2017. Available at: https://www.cdc. gov/cancer/hpv/statistics/race.htm. Accessed on 31 December 2020.

9. D'Souza G, Kreimer AR, Viscidi R, et al. Case-control study of human papillomavirus and oropharyngeal cancer. N Engl J Med 2007;356:1944-56.

10. Wong ML, Sen P, Wong CM, et al. Human immunodeficiency virus (HIV) prevention education in Singapore: challenges for the future. Ann Acad Med Singap 2012;41:602-9.

11. St Guily JL, Borget I, Vainchtock A, et al. Head and neck cancers in France: an analysis of the hospital medical information system (PMSI) database. Head Neck Oncol 2010;2:22.

12. Cancer network. FDA Approves Gardasil 9 for Prevention of Oropharyngeal, Head \& Neck Cancers Caused by HPV, 16 June 2020. Available at: https://www.cancernetwork.com/view/fda-approvesgardasil-9-for-prevention-of-oropharyngeal-head-and-neck-cancerscaused-by-hpv. Accessed on 31 December 2020.

13. Ministry of Health, Singapore. Nationally Recommended Vaccines. Available at: https://www.moh.gov.sg/resources-statistics/nationallyrecommended-vaccines. Accessed on 31 December 2020.

Ernest Wei Zhong $\underline{\mathrm{Fu}}^{1}{ }^{1}{ }_{\text {MRC (Edinburgh), }}$

Nandini Chilagondanahalli ${ }^{2} M D$,

Manish Mahadeorao Bundele ${ }^{2}$ FRCPath,

Jereme Yijin Gan ${ }^{1}$ MRCS (Edinburgh),

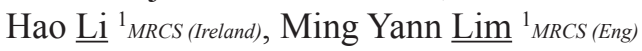

\footnotetext{
1 Department of Otorhinolaryngology, Head and Neck Surgery, Tan Tock Seng Hospital, Singapore

${ }^{2}$ Department of Pathology, Tan Tock Seng Hospital, Singapore
}

Correspondence: Dr Ernest Wei Zhong Fu, Department of Otorhinolaryngology, Head and Neck Surgery, Tan Tock Seng Hospital, 11 Jalan Tan Tock Seng, Singapore 308433.

Email: ernest_wz_fu@ttsh.com.sg 